doi: $10.2306 /$ scienceasia1513-1874.2014.40.451

\title{
On the solutions and conservation laws for the Sharma-Tasso-Olver equation
}

\author{
Andrew Gratien Johnpillai ${ }^{\mathrm{a}}$, Chaudry Masood Khalique $^{\mathrm{b}, *}$ \\ a Department of Mathematics, Eastern University, 30350, Sri Lanka \\ b International Institute for Symmetry Analysis and Mathematical Modelling, \\ Department of Mathematical Sciences, North-West University, Mafikeng Campus, Private Bag X 2046, \\ Mmabatho 2735, South Africa
}

*Corresponding author, e-mail: Masood.Khalique@nwu.ac.za

Received 3 Apr 2014

Accepted 28 Sep 2014

\begin{abstract}
We study the Sharma-Tasso-Olver equation from the Lie symmetry point of view. We derive the Lie point symmetry generators of the equation and classify them to obtain the optimal system of one-dimensional subalgebras of the Lie symmetry algebra of the equation. These subalgebras are then used to construct symmetry reductions for the equation. We obtain the general solution of the nonlinear second-order ordinary differential equation which results from the symmetry reduction for the travelling wave group-invariant solutions of the equation by transforming it into a linear third-order ordinary differential equation through a Riccati transformation. Then we show that one can easily obtain the travelling wave exact group-invariant solutions for the underlying equation by using the general solution of the linearized third-order ordinary differential equation and the Riccati transformation. We also construct conservation laws for the underlying equation by making use of the multiplier method.
\end{abstract}

KEYWORDS: Lie point symmetries, optimal system, group-invariant solutions, multiplier method

\section{INTRODUCTION}

Nonlinear evolution equations (NLEEs) appear in many branches of applied mathematics, physics, and engineering. The investigation of exact solutions of NLEEs plays an important role in the study of these equations. One of the most widely studied aspects is their integrability since the existence of the exact solutions enables one to have a better understanding of the phenomena modelled by these NLEEs. Moreover, the existence of such exact solutions to these NLEEs facilitates the verification of numerical methods and helps in the stability analysis of solutions. Also, the study of existence of conservation laws of partial differential equations (PDEs) further confirms their integrability and plays an important role in the solution process of PDEs.

In the past few decades, because of the increased interest for finding the exact solutions, a variety of analytical and computational methods have been developed for the analysis of these NLEEs, for example, the Adomian decomposition method, the inverse scattering transform, the Darboux transformation method, the Hirota bilinear method, the Lie-Bäcklund transformation method, the $\left(G^{\prime} / G\right)$-expansion method, the exponential function method, the $F$-expansion method and many others.

The Sharma-Tasso-Olver (STO) equation has the form

$$
u_{t}+3 \alpha u_{x}^{2}+3 \alpha u^{2} u_{x}+3 \alpha u u_{x x}+\alpha u_{x x x}=0 .
$$

In (1), $\alpha$ is an arbitrary real constant. The STO equation (1) appears in many scientific applications such as in quantum field theory, relativistic physics, dispersive wave phenomena, plasma physics, nonlinear optics, and applied and physical sciences ${ }^{1-3}$. Many authors have studied the STO equation (1) in recent years because of its applications in several areas of interest (see Refs. 4-6 and the references therein).

The transformation

$$
\tilde{t}=t / \alpha, \quad \tilde{x}=x, \quad \tilde{u}=u
$$

maps (1) to the PDE

$$
\tilde{u}_{\tilde{t}}+3 \tilde{u}_{\tilde{x}}^{2}+3 \tilde{u}^{2} \tilde{u}_{\tilde{x}}+3 \tilde{u} \tilde{u}_{\tilde{x} \tilde{x}}+\tilde{u}_{\tilde{x} \tilde{x} \tilde{x}}=0 .
$$

Hence, without loss of generality, dropping the tildes in (3), we can consider the PDE of the form

$$
u_{t}+3 u_{x}^{2}+3 u^{2} u_{x}+3 u u_{x x}+u_{x x x}=0
$$

in our analysis as all the results of (4) can easily be extended to the class (1) by the transformation (2). 
In this paper, the method of Lie point symmetry and multiplier approach will be used to study the exact solutions and conservation laws for the STO equation (4). In Ref. 7, the authors have investigated the potential form of (1) for exact solutions using the potential symmetry method.

During the past four decades, Lie symmetry analysis has proved to be a powerful tool for solving a given nonlinear system of PDEs in a systematic manner. For the theory and application of the Lie symmetry methods, see, e.g., Refs. 8-10.

Here we use the Lie symmetry method to obtain all the Lie point symmetries of (4). It is found that (4) admits a three-dimensional Lie algebra. Then with the help of these Lie point symmetries we construct the optimal system of one-dimensional subalgebras of the Lie algebra of (4). We obtain symmetry reductions using these optimal system of one-dimensional subalgebras. In the process to construct travelling wave group-invariant solutions for (4) we have found that the nonlinear second-order ordinary differential equation (ODE), which is a consequence of symmetry reduction, is linearizable to a third-order ODE through a Riccati transformation ${ }^{11,12}$. By integrating the linearized third-order ODE and using the Riccati transformation again we obtain the travelling wave exact group-invariant solutions for (4). It should be mentioned that our analysis of travelling wave exact solutions of the STO equation (1) is much simpler than those mentioned in Refs. 3-6. We also construct conservation laws for (4) by making use of the multiplier method $^{10,13,14}$.

The outline of the paper is as follows. First we present the main operators and briefly discuss the approach in the construction of the conservation laws for (4). The Lie point symmetries of (4) are then obtained and used to construct the optimal system of one-dimensional subalgebras of the Lie algebra of (4). Symmetry reductions and exact solutions of (4) are derived by using the optimal system of one-dimensional subalgebras. We then construct conservation laws for (4) via the multiplier method.

\section{PRELIMINARIES}

In this section, we provide the notation and some important results. For details the reader is referred to Refs. 9,13, 14.

Consider a $k$ th-order system of PDEs of $n$ independent variables $x=\left(x^{1}, x^{2}, \ldots, x^{n}\right)$ and $m$ dependent variables $u=\left(u^{1}, u^{2}, \ldots, u^{m}\right)$, namely

$$
E_{\alpha}\left(x, u, u_{(1)}, \ldots, u_{(k)}\right)=0, \quad \alpha=1, \ldots, m,
$$

where $u_{(1)}, u_{(2)}, \ldots, u_{(k)}$ denote the collections of all first, second, ..., $k$ th-order partial derivatives, that is, $u_{i}^{\alpha}=D_{i}\left(u^{\alpha}\right), u_{i j}^{\alpha}=D_{j} D_{i}\left(u^{\alpha}\right), \ldots$, respectively, with the total derivative operator with respect to $x^{i}$ given by

$$
D_{i}=\frac{\partial}{\partial x^{i}}+u_{i}^{\alpha} \frac{\partial}{\partial u^{\alpha}}+u_{i j}^{\alpha} \frac{\partial}{\partial u_{j}^{\alpha}}+\ldots, \quad i=1, \ldots, n,
$$

where the summation convention is used whenever appropriate.

A Lie-Bäcklund operator is given by

$$
X=\xi^{i} \frac{\partial}{\partial x^{i}}+\eta^{\alpha} \frac{\partial}{\partial u^{\alpha}}+\sum_{s \geqslant 1} \zeta_{i_{1} i_{2} \ldots i_{s}}^{\alpha} \frac{\partial}{\partial u_{i_{1} i_{2} \ldots i_{s}}^{\alpha}},
$$

where $\xi^{i}, \eta^{\alpha} \in \mathcal{A}$ and $\mathcal{A}$ is the space of differential functions. The additional coefficients $\zeta_{i_{1} i_{2} \ldots i_{s}}^{\alpha}$ are determined uniquely by the prolongation formulae

$$
\begin{aligned}
\zeta_{i}^{\alpha} & =D_{i}\left(W^{\alpha}\right)+\xi^{j} u_{i j}^{\alpha}, \\
\zeta_{i_{1} \ldots i_{s}}^{\alpha} & =D_{i_{1}} \ldots D_{i_{s}}\left(W^{\alpha}\right)+\xi^{j} u_{j i_{1} \ldots i_{s}}^{\alpha}, \quad s>1,
\end{aligned}
$$

in which $W^{\alpha}=\eta^{\alpha}-\xi^{j} u_{j}^{\alpha}$ is the Lie characteristic function.

The $n$-tuple vector $T=\left(T^{1}, T^{2}, \ldots, T^{n}\right)$ where $T^{j} \in \mathcal{A}$ and $j=1, \ldots, n$ is a conserved vector of (5) if $T^{i}$ satisfies

$$
D_{i} T^{i}=0
$$

on (5). Equation (8) is called a local conservation law of system (5).

It can be shown that every admitted conservation law arises from multipliers $Q^{\alpha}\left(x, u, u_{(1)}, \ldots\right)$ such that

$$
Q^{\alpha} E_{\alpha}=D_{i} T^{i}
$$

holds identically ${ }^{10,13}$. In the multiplier approach for conservation laws, one takes the variational derivative of (9), that is,

$$
\frac{\delta}{\delta u^{\beta}}\left(Q^{\alpha} E_{\alpha}\right)=0
$$

holds for arbitrary functions of $u\left(x^{1}, x^{2}, \ldots, x^{n}\right)$, (see also Refs. 10,14). All the multipliers can be derived from the determining equation (10) for which the underlying equation is expressed as a local conservation law.

\section{LIE POINT SYMMETRY GENERATORS}

A vector field

$$
X=\tau(t, x, u) \frac{\partial}{\partial t}+\xi(t, x, u) \frac{\partial}{\partial x}+\eta(t, x, u) \frac{\partial}{\partial u}
$$


is a generator of a point symmetry of (4) if

$$
X^{[3]}\left[u_{t}+3 u_{x}^{2}+3 u^{2} u_{x}+3 u_{x} u_{x x}+u_{x x x}\right]=0 .
$$

on (4). Here the operator $X^{[3]}$ is the third prolongation of the operator $X$ and is defined by

$$
X^{[3]}=X+\zeta_{t} \frac{\partial}{\partial u_{t}}+\zeta_{x} \frac{\partial}{\partial u_{x}}+\zeta_{x x} \frac{\partial}{\partial u_{x x}}+\zeta_{x x x} \frac{\partial}{\partial u_{x x x}},
$$

where the coefficients $\zeta_{t}, \zeta_{x}, \zeta_{x x}$ and $\zeta_{x x x}$ are given by

$$
\begin{aligned}
\zeta_{t} & =D_{t}(\eta)-u_{t} D_{t}(\tau)-u_{x} D_{t}(\xi), \\
\zeta_{x} & =D_{x}(\eta)-u_{t} D_{x}(\tau)-u_{x} D_{x}(\xi), \\
\zeta_{x x} & =D_{x}\left(\zeta_{x}\right)-u_{x t} D_{x}(\tau)-u_{x x} D_{x}(\xi), \\
\zeta_{x x x} & =D_{x}\left(\zeta_{x x}\right)-u_{x x t} D_{x}(\tau)-u_{x x x} D_{x}(\xi) .
\end{aligned}
$$

The operator $D_{i}$ is the total derivative operator as given in (6).

The infinitesimal coefficients $\tau, \xi$ and $\eta$ are independent of the derivatives of $u$. Thus by equating the coefficients of like derivatives of $u$ in the determining equation (12) we obtain the following overdetermined system of linear PDEs:

$$
\begin{array}{r}
\tau=\tau(t), \\
\xi=\xi(t, x), \\
\eta_{u u}=0, \\
\tau_{t}-3 \xi_{x}=0, \\
\tau_{t}-2 \xi_{x}+\eta_{u}=0, \\
\eta-2 u \xi_{x}+u \tau_{t}-\xi_{x x}+\eta_{x u}=0, \\
\eta_{t}+3 u^{2} \eta_{x}+3 u \eta_{x x}+\eta_{x x x}=0, \\
6 u \eta-\xi_{t}-3 u^{2} \xi_{x}+3 u^{2} \tau_{t}+6 \eta_{x} \\
-3 u \xi_{x x}+6 u \eta_{x u}-\xi_{x x x}+3 \eta_{x x u}=0 .
\end{array}
$$

Solving the above determining equations for the coefficients $\tau, \xi$ and $\eta$, we obtain the following three Lie point symmetry generators admitted by (4):

$$
X_{1}=\frac{\partial}{\partial t}, X_{2}=\frac{\partial}{\partial x}, X_{3}=3 t \frac{\partial}{\partial t}+3 x \frac{\partial}{\partial x}-u \frac{\partial}{\partial u} \text {. }
$$

\section{OPTIMAL SYSTEM OF ONE-DIMENSIONAL SUBALGEBRAS OF THE LIE ALGEBRA}

In order to derive reductions and to construct classes of group-invariant solutions for (4) in a systematic manner, we obtain an optimal system of onedimensional subalgebras for (4). We will use the method for constructing an optimal system of onedimensional subalgebras as given in Ref. 10 .
Table 1 The adjoint table for the symmetries.

\begin{tabular}{cccc}
\hline Ad & $X_{1}$ & $X_{2}$ & $X_{3}$ \\
\hline$X_{1}$ & $X_{1}$ & $X_{2}$ & $X_{3}-3 \varepsilon X_{1}$ \\
$X_{2}$ & $X_{1}$ & $X_{2}$ & $X_{3}-\varepsilon X_{2}$ \\
$X_{3}$ & $\exp (3 \varepsilon) X_{1}$ & $\exp (3 \varepsilon) X_{2}$ & $X_{3}$ \\
\hline
\end{tabular}

To calculate the adjoint representation, we use the following well-known Lie series:

$$
\begin{aligned}
\operatorname{Ad}(\exp (\varepsilon X)) Y= & Y-\varepsilon[X, Y]+\frac{1}{2 !} \varepsilon^{2}[X,[X, Y]] \\
& -\frac{1}{3 !} \varepsilon^{3}[X,[X,[X, Y]]+\cdots,
\end{aligned}
$$

where Ad means the adjoint representation of a Lie group on its Lie algebra, together with the commutation relations of the three Lie symmetry generators (13) which are $\left[X_{1}, X_{2}\right]=0,\left[X_{1}, X_{3}\right]=$ $3 X_{1},\left[X_{2}, X_{3}\right]=X_{2}$.

For example,

$$
\begin{array}{r}
\operatorname{Ad}\left(\exp \left(\varepsilon X_{1}\right)\right) X_{3}=X_{3}-\varepsilon\left[X_{1}, X_{3}\right] \\
+\frac{1}{2 !} \varepsilon^{2}\left[X_{1},\left[X_{1}, X_{3}\right]\right]-\cdots \\
=X_{3}-3 \varepsilon X_{1} .
\end{array}
$$

Similarly, we can find the other entries of the adjoint table. We thus have the adjoint representation given by Table 1 .

In Table 1 , the $(i, j)$ entry represents $\operatorname{Ad}\left(\exp \left(\varepsilon X_{i}\right)\right) X_{j}$. For a non-zero vector

$$
X=a_{1} X_{1}+a_{2} X_{2}+a_{3} X_{3}
$$

we have to simplify the coefficients $a_{i}$ as far as possible through adjoint maps to $X$. The calculations are easy and we obtain an optimal system of onedimensional subalgebras spanned by $X_{2}, X_{1}+\varepsilon X_{2}$, and $X_{3}$, where $\varepsilon=0, \pm 1$.

\section{REDUCTION AND GROUP-INVARIANT SOLUTIONS}

In this section we use the optimal system of onedimensional subalgebras of the Lie algebra of (4), found above, to construct symmetry reductions and exact group-invariant solutions for (4).

(a) $X_{2}$. The group-invariant solution corresponding to $X_{2}$ is $u=h(\gamma)$, where $\gamma=t$ is the group invariant of $X_{2}$. Substituting this solution into (4) and solving the ODE, we obtain the solution for (4) given by $u(t, x)=C$, where $C$ is a constant.

(b) $X_{1}+\varepsilon X_{2}$, where $\varepsilon=0, \pm 1$ (travelling wave solutions). Here, the group-invariant solution is given 
by $u(t, x)=h(\gamma)$, where $\gamma=x-\varepsilon t$ is the group invariant of $X_{1}+\varepsilon X_{2}$. Substitution of this solution into (4) gives

$$
h^{\prime \prime \prime}+3 h h^{\prime \prime}+3 h^{2} h^{\prime}+3 h^{\prime 2}-\varepsilon h^{\prime}=0,
$$

where the prime denotes differentiation with respect to $\gamma$. Integrating (15) with respect to $\gamma$, we obtain

$$
h^{\prime \prime}+3 h h^{\prime}+h^{3}-\varepsilon h=C,
$$

where $C$ is an arbitrary constant of integration. We find that through the Riccati transformation

$$
h(\gamma)=\frac{v^{\prime}}{v}
$$

where $v^{\prime}=\mathrm{d} v / \mathrm{d} \gamma$, the ODE (16) can be linearized to a third-order ODE of the form

$$
v^{\prime \prime \prime}-\varepsilon v^{\prime}-C v=0 .
$$

The characteristic equation $m^{3}-\varepsilon m-C=0$ of (18) has roots $m_{i}, i=1,2,3$ given by

$$
\begin{gathered}
m_{1}^{3}-\varepsilon m_{1}-C=0, \\
m_{2,3}=\frac{-m_{1} \pm \sqrt{-3 m_{1}^{2}+4 \varepsilon}}{2} .
\end{gathered}
$$

Three cases arise.

Case $1\left(m_{1} \neq m_{2} \neq m_{3}\right)$. In this case, the general solution of (18) is given by

$$
v=C_{1} \exp \left(m_{1} \gamma\right)+C_{2} \exp \left(m_{2} \gamma\right)+C_{3} \exp \left(m_{3} \gamma\right)
$$

where $C_{1}, C_{2}$ and $C_{3}$ are arbitrary constants. Thus the general solution of (16), using (17), takes the form

$$
h(\gamma)=\frac{m_{1} \mathrm{e}^{m_{1} \gamma}+m_{2} A_{1} \mathrm{e}^{m_{2} \gamma}+m_{3} A_{2} \mathrm{e}^{m_{3} \gamma}}{\mathrm{e}^{m_{1} \gamma}+A_{1} \mathrm{e}^{m_{2} \gamma}+A_{2} \mathrm{e}^{m_{3} \gamma}}
$$

where $A_{1}=C_{2} / C_{1}$ and $A_{2}=C_{3} / C_{1}$. Hence the group-invariant solution of (4) is given by

$$
u(t, x)=\frac{m_{1} \mathrm{e}^{m_{1} \gamma}+m_{2} A_{1} \mathrm{e}^{m_{2} \gamma}+m_{3} A_{2} \mathrm{e}^{m_{3} \gamma}}{\mathrm{e}^{m_{1} \gamma}+A_{1} \mathrm{e}^{m_{2} \gamma}+A_{2} \mathrm{e}^{m_{3} \gamma}},
$$

where $\gamma=x-\varepsilon t$.

Case $2\left(m_{1}=m_{2} \neq m_{3}\right)$. Here the general solution to (18) takes the form

$$
v=\left(C_{1}+C_{2} \gamma\right) \exp \left(m_{1} \gamma\right)+C_{3} \exp \left(m_{3} \gamma\right),
$$

where $C_{1}, C_{2}$ and $C_{3}$ are arbitrary constants. Thus the group-invariant solution of (4) using (17) is given by

$$
\begin{aligned}
& u(t, x) \\
& =\frac{A_{1} \mathrm{e}^{m_{1} \gamma}+m_{1}\left[1+A_{1} \gamma\right] \mathrm{e}^{m_{1} \gamma}+m_{3} A_{2} \mathrm{e}^{m_{3} \gamma}}{\left[1+A_{1} \gamma\right] \mathrm{e}^{m_{1} \gamma}+A_{2} \mathrm{e}^{m_{3} \gamma}},
\end{aligned}
$$

where $\gamma=x-\varepsilon t, A_{1}=C_{2} / C_{1}$ and $A_{2}=C_{3} / C_{1}$.

Case $3\left(m_{1}=m_{2}=m_{3}\right)$. Here we find that the general solution to (18) can be given in the form

$$
v=\left(C_{1}+C_{2} \gamma+C_{3} \gamma^{2}\right) \exp \left(m_{1} \gamma\right),
$$

where $C_{1}, C_{2}$ and $C_{3}$ are arbitrary constants. Thus the group-invariant solution of (4) using (17) is given by

$$
u(t, x)=\frac{\left\{A_{1}+2 A_{2} \gamma+m_{1}\left[1+A_{1} \gamma+A_{2} \gamma^{2}\right]\right\}}{\left[1+A_{1} \gamma+A_{2} \gamma^{2}\right]},
$$

where $\gamma=x-\varepsilon t, A_{1}=C_{2} / C_{1}$, and $A_{2}=C_{3} / C_{1}$.

(c) $X_{3}$. This symmetry generator gives rise to the group-invariant solution $u(t, x)=t^{-1 / 3} h(\gamma)$, where $\gamma=x t^{-1 / 3}$ is the group invariant of $X_{3}$. Substitution of this solution into (4) results in the ODE

$$
3 h^{\prime \prime \prime}+9 h h^{\prime \prime}+9 h^{2} h^{\prime}+9 h^{\prime 2}-\gamma h^{\prime}-h=0 .
$$

\section{CONSERVATION LAWS}

In this section, we construct conservation laws for (4) using the multiplier approach as outlined above.

We consider the multipliers of the form $Q\left(t, x, u, u_{t}, u_{x}\right)$ for (4). The determining equation (10) for the multipliers takes the form

$$
\frac{\delta}{\delta u}\left[Q\left(u_{t}+3 u_{x}^{2}+3 u^{2} u_{x}+3 u_{x} u_{x x}+u_{x x x}\right)\right]=0 .
$$

Expanding (29) yields

$$
\begin{array}{r}
Q_{u}\left[u_{t}+3 u_{x}^{2}+3 u^{2} u_{x}+3 u_{x} u_{x x}+u_{x x x}\right] \\
-D_{t}\left[Q_{u_{t}}\left(u_{t}+3 u_{x}^{2}+3 u^{2} u_{x}+3 u_{x} u_{x x}+u_{x x x}\right)\right] \\
-D_{x}\left[Q_{u_{x}}\left(u_{t}+3 u_{x}^{2}+3 u^{2} u_{x}+3 u_{x} u_{x x}+u_{x x x}\right)\right] \\
+Q\left(6 u u_{x}+3 u_{x x}\right)-D_{t}(Q)-D_{x}\left[Q\left(6 u_{x}+3 u^{2}\right)\right] \\
+D_{x}^{2}(3 u Q)-D_{x}^{3}(Q)=0 .
\end{array}
$$

In (30), we equate the coefficients of the derivatives of $u$ to zero and obtain an overdetermined system of linear PDEs. Solving this system we find that the multiplier $Q$ takes the form

$$
Q=c_{1},
$$


where $c_{1}$ is a constant. The conserved vector $\left(T^{1}, T^{2}\right)$ of (4) satisfies the divergence relation given by

$$
\begin{array}{r}
Q\left[u_{t}+3 u_{x}^{2}+3 u^{2} u_{x}+3 u_{x} u_{x x}+u_{x x x}\right] \\
=D_{t} T^{1}+D_{x} T^{2},
\end{array}
$$

for all arbitrary functions $u(t, x)$. From (31) and (32), we have

$$
\begin{aligned}
& c_{1}\left[u_{t}+3 u_{x}^{2}+3 u^{2} u_{x}+3 u_{x} u_{x x}+u_{x x x}\right] \\
& \quad=D_{t}\left(c_{1} u\right)+D_{x}\left[c_{1}\left(u^{3}+3 u u_{x}+u_{x x}\right)\right] .
\end{aligned}
$$

Thus whenever $u(t, x)$ is solution of (4), we have

$$
D_{t}\left(c_{1} u\right)+D_{x}\left[c_{1}\left(u^{3}+3 u u_{x}+u_{x x}\right)\right]=0 .
$$

Hence we derive the following conserved vector for (4) from (34):

$$
T^{1}=u, \quad T^{2}=u^{3}+3 u u_{x}+u_{x x} .
$$

\section{CONCLUDING REMARKS}

We mention here that this approach to construct the travelling wave group-invariant solutions of (4) has not been reported earlier. Furthermore, since (4) is an evolution equation and of odd order, it is not variational. We therefore applied the multiplier method to find its conservation laws. We found that for (4), this method gives rise to one multiplier and thus one conserved vector is obtained.

\section{REFERENCES}

1. Ugurlu Y, Kaya D (2007) Analytic method for solitary solutions of some partial differential equations. Phys Lett A 370, 251-9.

2. Bekir A, Boz A (2008) Exact solutions for nonlinear evolution equation using Exp-function method. Phys Lett A 372, 1619-25.

3. Pan JT, Chen WZ (2009) A new auxilliary equation method and its application to the Sharma-Tasso-Olver equation. Phys Lett A 373, 3118-21.

4. Yan Z (2003) Integrability of two types of the (2+1)dimensional generalized Sharma-Tasso-Olver integrodifferential equations. MM Research Preprints, No. 22, pp 302-24.

5. Wazwaz AM (2007) New solitons and kinks solutions to the Sharma-Tasso-Olver equation. Appl Math Comput 188, 1205-13.

6. He Y, Li S, Long Y (2013) Exact solutions to the Sharma-Tasso-Olver equation by using improved $G^{\prime} / G$-expansion method. J Appl Math 2013, 247234.

7. Lian ZJ, Lou SY (2005) Symmetries and exact solutions of the Sharma-Tass-Olver equation. Nonlin Anal 63, 1167-77.
8. Bluman GW, Kumei S (1989) Symmetries and Differential Equations, Springer Verlag, New York, NY. USA.

9. Ibragimov NH (1994-1996) CRC Handbook of Lie Group Analysis of Differential Equations, Vol 1-3, CRC Press, Boca Raton, FL.

10. Olver PJ (1993) Applications of Lie Groups to Differential Equations, 2nd edn, Springer Verlag, New York, NY, USA.

11. Johnpillai AG, Khalique CM, Mahomed FM (2012) Lie and Riccati linearization of a class of Liénard type equations. J Appl Math 2012, 171205.

12. Pandey SN, Lakshmanan M (2005) Lie symmetries and linearization of a generalized modified Emden type equation. In: Proceedings of the 2nd National Conference on Nonlinear Systems and Dynamics, pp 1-4.

13. Steudel H (1962) Über die Zuordnung zwischen Invarianzeigenschaften und Erhaltungssatzen. $Z$ Naturforsch 17A, 129-32.

14. Anco SC, Bluman GW (2002) Direct construction method for conservation laws of partial differential equations, Part I: Examples of conservation law classifications. Eur J Appl Math 13, 545-66. 